\title{
Instrumental Improvements for Rapid, Detailed Kinetic Studies of Oxygenase Activity
}

\author{
Harold W. Cook, ${ }^{1}$ Gordon Ford, and William E. M. Lands \\ Department of Biological Chemistry, The University of Michigan, Ann Arbor, Michigan 48109
}

Received January 10, 1979

\begin{abstract}
The characteristics of an electronic differentiator that facilitates continuous monitoring of changes in the oxygen concentration measured by a polarographic oxygen electrode are described. In addition, some properties of the electrode membranes that increase the sensitivity of the oxygen-sensing electrode are also described. These modifications greatly improve the accuracy and sensitivity of the biological oxygen monitor for use in kinetic studies of complex enzyme systems such as soybean lipoxygenase or prostaglandin cyclooxygenase. The electronic differentiator also may be used in conjunction with other ratemonitoring instruments such as spectrophotometers and $\mathrm{pH}$ meters for accurate quantitation of rapidly changing rates.
\end{abstract}

Since the measurement of oxygen tensions in blood and tissues using a polarographic method was first described by Clark (1), numerous modifications to the oxygenmonitoring electrode and various application of this technique have been reported (e.g., Refs. 2-8). One very useful experimental application of the oxygen electrode has been to the measurement of $\mathrm{O}_{2}$ consumption or production during enzymecatalyzed reactions; changes in rates and the total extent of reactions can be determined from continuous monitoring of the electrode signal. For such applications the oxygen electrode is sensitive in a broad range of solutions where photometric procedures for oxygen determination may be complex or inadequate.

As an improvement on our approach to studies of the kinetics and mechanisms of fatty acid oxygenation by prostaglandin cyclooxygenase $(9-12)$ and soybean lipoxygenase $(13,14)$, we have modified a com-

\footnotetext{
${ }^{1}$ Present Address: Departments of Pediatrics and Biochemistry, Dalhousie University. Halifax, Nova Scotia, B3H 4H7. Author to whom all correspondence should be addressed.
}

mercially available biological oxygen-electrode system to optimize measurement of oxygen consumption by various enzyme preparations under a variety of experimental conditions. An electronic differentiator that instantaneously quantitates changes in oxygen concentration of reaction mixtures has been constructed to facilitate evaluation of change in reaction rates that might not be observed with less attention to each phase of the enzymatic reaction. We now describe in detail the characteristics of our electronic differentiator as well as some modification to the oxygen electrode that increase the sensitivity and versatility of this method of measurement of enzyme activities. The electronic differentiator that we describe also can be used with other rate-monitoring instruments such as spectrophotometers and $\mathrm{pH}$ meters for accurate quantitation of rapidly changing rates.

\section{MATERIALS AND METHODS}

Biological oxygen monitor. Changes in oxygen concentration were monitored with a Yellow Springs Instrument Company Model 53 oxygen monitor that had been 
adapted to provide sequential monitoring of four different electrodes. The standard bath assembly was equipped with a variablespeed magnetic stirrer capable of a range of approximately 200 to $3000 \mathrm{rpm}$, and a regulated temperature $\left(30 \pm 0.5^{\circ} \mathrm{C}\right)$ was maintained with a Haake (Berlin, West Germany) constant temperature circulator. A second output terminal was added to the oxygen monitor to bypass the recorder output attenuator. This gave an output to the electronic differentiator of approximately $1.0 \mathrm{~V}$ for a $100 \%$ (full scale) value on the meter of the monitor.

Electronic differentiator. The differentiator was developed to detect and quantitate slow rates from the biological oxygen monitor in the presence of both high frequency noise and baseline drift. The block diagram shown in Fig. 1 indicates the relationships of the various components. The timing module indicated in the lower left controls the sampling interval and regulates the sequence of manipulations performed on the signal. The noise reject circuit $\left(A_{2}-A_{3}\right)$, including a dead band circuit, was put ahead of the main differentiator stage $\left(\mathrm{A}_{4}\right)$ to reduce the effects of small changes and slow drift in the baseline. Since the dead band circuit must work against a zero signal (or power supply common), and since the $\mathrm{O}_{2}$ monitor typically has a baseline of 20 to
$100 \mathrm{mV}$, the circuit had to be altered. Introduction of an offset to cancel out the baseline voltage was impractical because of the variable nature of the baseline voltage.

To overcome this difficulty, the input circuit was developed as indicated in the detailed schematic in Fig. 2a. Amplifier $A_{1}$ is an instrumentation amplifier with variable gain. The electrode input signal is introduced through the inverting $(-)$ input to this amplifier. The noninverting (t) input to $A_{1}$ provides the balancing voltage. When $A_{1}$ detects a voltage difference between the two inputs, its output reflects the voltage times an attenuation factor which has been adjusted by the setting of the noise attenuation control. The resultant output is fed to the conventional dead band circuit (diode resistor network).

If the output voltage of $A_{1}$ is greater than the biasing of the dead band circuit, it appears at the noninverting (+) input of the isolation amplifier $A_{3}$, a unity gain follower, and at the inverting $(-)$ input of the integrator $\left(A_{2}\right)$, which integrates for a fixed 0.1 -s interval. The smoothed output of $A_{2}$ goes to the noninverting (+) input of $A_{1}$. As this reference voltage from $A_{2}$ approaches the level of the signal ( - ) input voltage to $A_{1}$, the difference approaches zero and the output voltage of $A_{1}$ falls to zero, which in turn causes the integrator $A_{2}$ to come to a

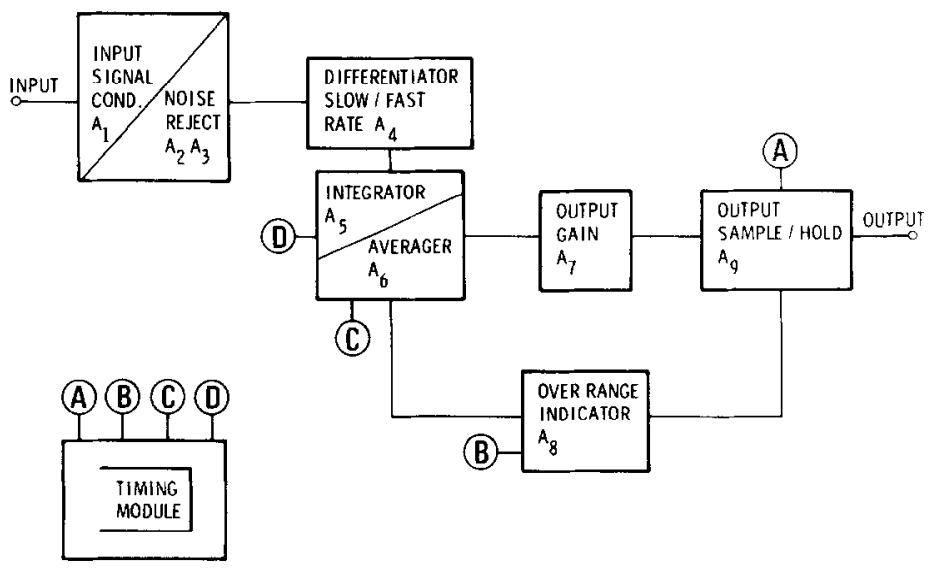

FIG. 1. General schematic of reaction monitor differentiator. 
steady state. The output voltage of $A_{2}$ at this time is equal to the $(-)$ input voltage of $A_{1}$.

The output of $A_{2}$ is also fed to the differentiator, $A_{4}$, as a relatively clean signal since it has been conditioned as described above. The input to the differentiator operational amplifier is fitted with two different capacitors to provide for fast and slow reaction rates, allowing the slower signals to be multiplied 10-fold to improve signal-tonoise ratios. Unfortunately, the uutput of the differentiator amplifier $\left(A_{4}\right)$ still has some noise, due partly to internal amplifier noise and partly to power supply noise. Although of a very low level, this noise can still obscure some of the very slow rates that have been sufficiently amplified through the $10 \mathrm{X}$ circuit.

Amplifier $A_{5}$, was connected as an integrator (see Fig. 2b) with variable intervals so that several output periods determined by the timing module could be averaged. Amplifier $A_{6}$ stores each output interval of $A_{5}$ and, to reduce sharp differences between adjacent 0.1 -s intervals, the present $\left(A_{5}\right)$ and previous $\left(A_{6}\right)$ signal intervals are sent to amplifier $A_{7}$ which averages these signals for further improvement of the signal-to-noise ratio. $A_{7}$ also serves as a variable gain $(\mathrm{X} 1, \mathrm{X} 10, \mathrm{X} 100)$ so that $A_{9}$, a digital sample/hold device, can be used to maximum resolution ( 12 bits). The digital sample/hold unit was used to eliminate output droop, should a long sampling period be used with very slow reactions.

The integrated circuit (I.C. $)$ is used as an overrange detector to indicate when the input of $A_{9}$ is overloaded and that a lower output level from $A_{7}$ should be selected. $A_{8}$ is connected as a comparator, to indicate an overload at the input to $A_{7}$ and that the

$$
\frac{\text { recorder pen reading }}{100} \times\left(\frac{\text { recorder } \mathrm{mV} \text { range } \times \frac{60}{\text { sample interval }}}{\text { input amplification } \times \text { output amplification }}\right)=\mathrm{mV} / \mathrm{min} \text {. }
$$

slow-fast switch should be changed to the fast position. The output of $A_{9}$ to the recorder is scaled down from $\pm 5 \mathrm{~V}$ to \pm 100 $\mathrm{mV}$ to correspond to the normal output of the biological oxygen monitor. The timing module (Fig. 2c) generates the proper interval and time-delayed pulses so that the integrator, $A_{5}$, and sample/hold, $A_{9}$, function in the proper sequence for each sampling interval [(A) start conversion of $A_{9}$ before (C) resetting $A_{6}$ before (D) starting $A_{5}$ ].

Sudden alterations in electrode and differentiator response due to changes in line voltage were caused in part by other instruments on the same external circuit. To reduce this extraneous noise, a filter regulator, isolation transformer supplied by Topaz Electronics, San Diego, was incorporated into the main power-supply line.

Operation of the system. The differentiator-recorder system was calibrated on a dual-pen recorder with each recorder channel set for $100 \mathrm{mV}$ and the zero controls adjusted to set the pens to 0 . The $\mathrm{O}_{2}$ monitor was then adjusted for some convenient meter reading (e.g., 80\%) and the $\mathrm{O}_{2}$ monitor recorder output attenuator was adjusted so the recorder pen also indicated $80 \%$. The run/check switch of the differentiator assembly was turned to "check" and, at $1 X$ output and 1-s interval, the calibration control was adjusted so that the recorder pen from the differentiator read the same as that from the oxygen monitor output. The run/check switch was returned to "run" and the zero position checked at $100 \mathrm{X}$ output with the amp zero setting on the oxygen monitor and adjusting the differentiator zero control if needed. (It was necessary to go back and forth between these control settings several times.)

The differentiator is now calibrated to provide millivolts per minute as shown in the following equation: 

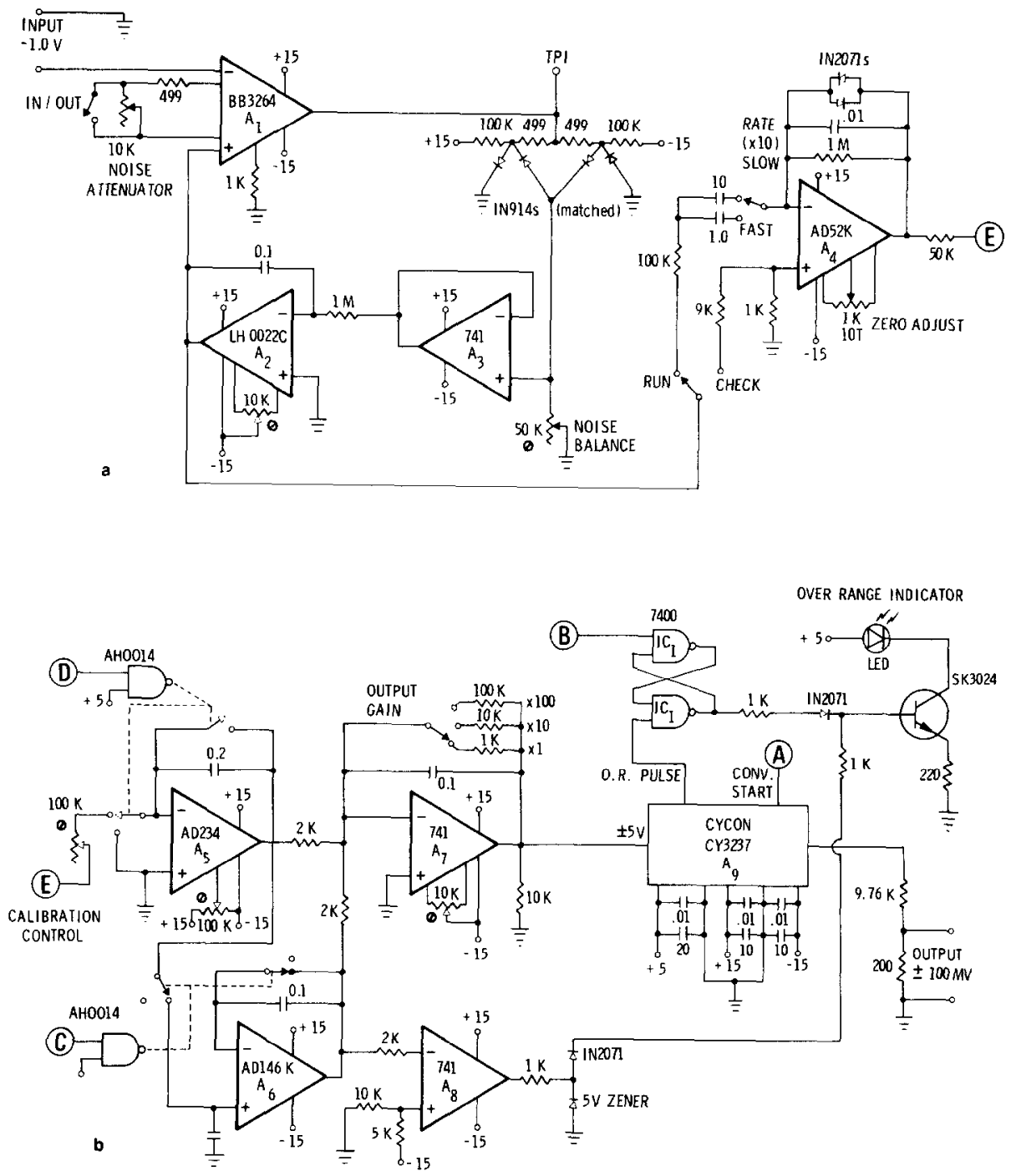

Fig. 2. Schematics of electronic component of the differentiator. (a) Signal conditioning and differentiation; (b) differentiator signal modulation; (c) timing module.

For example, with the recorder on $100-\mathrm{mV}$ range and the differentiator set at $0.1-\mathrm{s}$ interval, an input amplification of 10 and an output amplification of 100 gives an instrument factor of $(100 \times 60 / 0.1) /(10 \times 100)=60$. In this case, a $50 \%$ of full scale deflection of the pen represents a rate of $30 \mathrm{mV} / \mathrm{min}$.

Response of the differentiator was tested using a high-sensitivity function generator (Krohn-Hite Corporation, Cambridge, Mass., Model $5100 \mathrm{~A}$ ). The actual voltages presented to the differentiator assembly were independently recorded with the second pen of the dual-pen recorder (Leeds and Northrup Co., North Wales, Pa., Model EL-650). The recorded response could be attenuated by several adjustments which increase the instrument factor described above: (a) decreasing the time interval during which signal was accumulated by the differentiator, (b) changing the input rate amplification switch to "fast," (c) reducing 


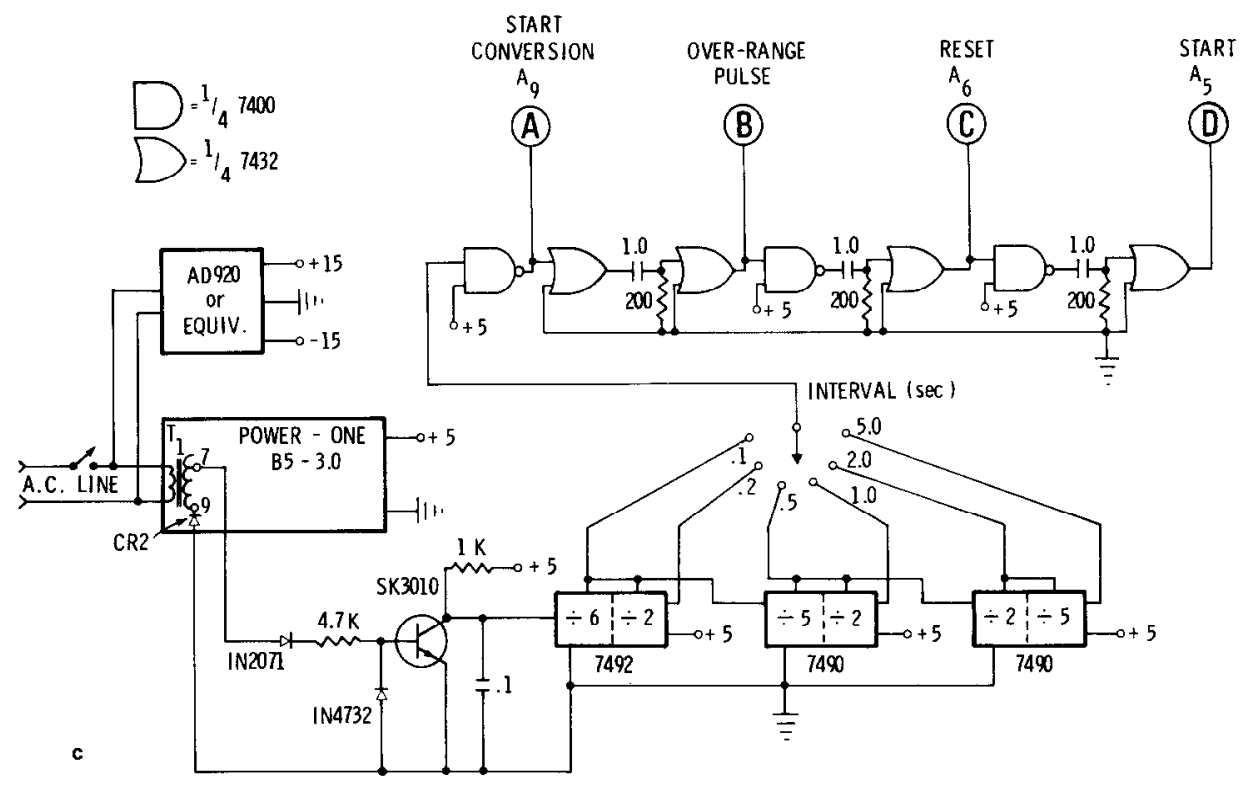

FIG. 2-Continued.

the output amplification, and (d) increasing the millivolts needed for full scale deflection of the recorder.

Standard membranes supplied for the oxygen electrodes were made of 12.5- or 25 - $\mu$ m-thick Teflon (Yellow Springs Instruments, Ltd.) We also examined methyl vinyl-silicone membranes, $12.5,25$, or 50 $\mu \mathrm{m}$ thick (Quantum, Inc., Wallingford, Conn.), and a dimethyl silicone membrane, $4.4 \mu \mathrm{m}$ thick (generously donated by R. L. Riley, Universal Oil Products Co., San Diego, Calif.).

\section{RESULTS}

\section{Quantitative Evaluation of the Electronic Differentiator}

When an instantaneous step function signal of $6 \mathrm{mV}$ was applied to the Electronic Differentiator approximately $2-3 \mathrm{~s}$ was required for the differentiator to return to a stable baseline. The recorded response of the differentiator was proportional to a constantly changing voltage signal as indicated in Fig. 3. Multiplying the deflection attained at steady state times the respective instrument factor gives $12.6,26,27$, and $55.5 \mathrm{mV} / \mathrm{min}$ corresponding to generated ramps of $13.5,27,27$, and $55 \mathrm{mV} / \mathrm{min}$, respectively. A wide range of changes in voltage with time could be monitored accurately as shown in Table 1 . The values obtained were accurate within $\pm 3 \%$ for a range of voltage changes of 3 to $50 \mathrm{mV} /$ min. Above $50 \mathrm{mV} / \mathrm{min}$, comparative evaluations of the response were limited by the accuracy of the assignment of the generated input from the signal generator. The generator could not accurately provide ramps slower than $3 \mathrm{mV} / \mathrm{min}$, but estimates of the noise levels in our system at maximal sensitivity indicated a signal-to-noise ratio of $3: 1$ would be obtained at input rates of 1 $\mathrm{mV} / \mathrm{min}$. Overall it is clear that this electronic arrangement can provide a reliable reflection of the rate of change in voltage from 0.5 to $1000 \mathrm{mV} / \mathrm{min}$.

Because we wished to study the action of fatty acid oxygenases which often exhibit both rapidly increasing and decreasing velocities, we tested the capacity of the differentiator to give the first derivative of a 


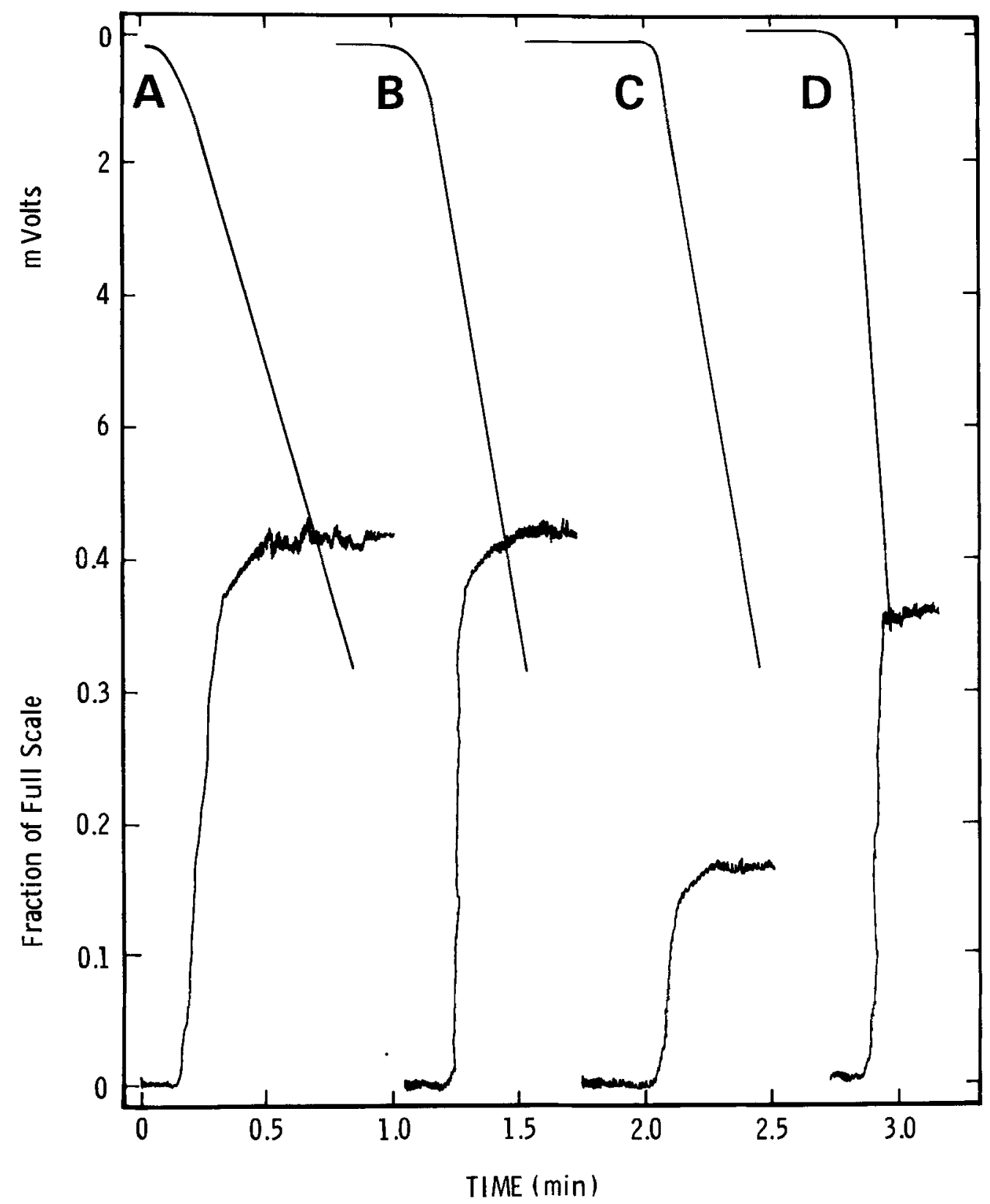

FIG. 3. Responses to changes in voltage by the electronic differentiator. An increasing voltage ramp indicated by the upper curves was generated by the function generator (see Materials and Methods). The recorded responses from the differentiator are shown in the lower curves for the respective instrument factors and input rate indicated. (A) $30,13.5 \mathrm{mV} / \mathrm{min}$; (B) $60,27 \mathrm{mV} / \mathrm{min}$; (C) $150,27 \mathrm{mV} / \mathrm{min}$; (D) $150,55 \mathrm{mV} / \mathrm{min}$.

continuously changing voltage input as illustrated in Fig. 4. Accurate derivatives were obtained for sine functions generated over a 200 -fold range of frequency and amplitude.
Thus the differentiator has the capacity to provide directly a value for the velocity at any time during a reaction with continuously changing velocity. 
TABLE 1

Agreement between InPut Rates and Rates Determined from the Differentiator-Recorder

\begin{tabular}{|c|c|c|c|c|c|}
\hline $\begin{array}{c}\text { Generated input } \\
\text { signal }(\mathrm{A}) \\
(\mathrm{mV} / \mathrm{min})\end{array}$ & $n$ & $\begin{array}{c}\text { Fractional } \\
\text { deflection } \\
\text { of recorder (B) }\end{array}$ & $\begin{array}{c}\text { Instrument } \\
\text { factor } \\
\text { (C) }\end{array}$ & $\begin{array}{l}\text { Output from } \\
\text { differentiator } \\
(\mathrm{B} \times \mathrm{C}) \\
(\mathrm{mV} / \mathrm{min})\end{array}$ & $\begin{array}{l}\text { Rate }_{(\text {in })} \\
\text { Rate }_{(\text {out })}\end{array}$ \\
\hline 3.4 & 2 & 0.230 & 15 & 3.4 & 1.00 \\
\hline 6.4 & 1 & 0.205 & 30 & 6.2 & 1.03 \\
\hline 12.8 & 2 & 0.415 & 30 & 12.5 & 1.02 \\
\hline 13.5 & 5 & 0.455 & 30 & 13.6 & 0.99 \\
\hline 12.8 & 1 & 0.206 & 60 & 12.4 & 1.03 \\
\hline 18.5 & 1 & 0.305 & 60 & 18.3 & 1.01 \\
\hline 25.0 & 2 & 0.415 & 60 & 24.9 & 1.00 \\
\hline 25.0 & 1 & 0.165 & 150 & 24.7 & 1.01 \\
\hline 52.0 & 4 & 0.352 & 150 & 52.8 & 0.98 \\
\hline 75.0 & 2 & 0.230 & 300 & 69 & 1.08 \\
\hline 150 & 1 & 0.465 & 300 & 139 & 1.07 \\
\hline 180 & 2 & 0.300 & 600 & 180 & 1.00 \\
\hline 340 & 1 & 0.520 & 600 & 312 & 1.09 \\
\hline 760 & 2 & 0.228 & 3000 & 684 & 1.11 \\
\hline
\end{tabular}

Some Characteristics of the OxygenSensing Electrodes

Since our principal use of the electronic differentiator was to evaluate changes in the rate of oxygen consumption with oxygensensing electrodes, we investigated some aspects of the electrode system to determine optimum operating conditions. When the auxiliary heater was used to oppose a coldwater thermal sink, distinct oscillations in electrode response were evident. This drift in the electrode signal in response to the very slight temperature changes was reduced by running the constant temperature bath at the most sensitive setting to maintain the reaction chamber at $30.0 \pm 0.5^{\circ} \mathrm{C}$. Less noise in the signal from the oxygen-sensing electrode was observed when the magnetic stirrer speed was maintained at 800-1000 rpm (compared to $400-600 \mathrm{rpm}$ by the commercial stirring device). At speeds greater than $1000 \mathrm{rpm}$, the magnets began to produce interference by bouncing against the membranes of the electrode tips.

The response to changes in oxygen concentrations of the reaction buffer as well as the variability between different electrodes was tested by oxidizing added NADH in the assay system described by Robinson and Cooper (15) (Table 2). With the electrodes in air-saturated buffer adjusted to $100 \%$ recorder deflection with the biological oxygen monitor on "air" range, an average deflection of $1 \mathrm{mV}$ per change of $2.34 \mu \mathrm{M} \mathrm{O}_{2}$ in the buffer was obtained. Thus it was determined that the air-saturated buffer contained $234 \mu \mathrm{M} \mathrm{O}_{2}$. The variability in the changes in oxygen concentration (determined directly from the oxygen electrode) and in changes in oxygen concentration with time (determined from the differentiator tracing) for several different electrodes was approximately $3 \%$.

The relative response of oxygen-sensing electrodes with different types of membranes was evaluated by very rapidly introducing 50,100 , or $200 \mu l$ of deaerated buffer, thereby decreasing the oxygen concentration in the chamber by $3.5,7$, or $14 \mu \mathrm{M} \mathrm{O}$. The electrode and differentiator responses were monitored with the recorder chart paper at maximum speed $(600 \mathrm{~cm} / \mathrm{h})$. Since with most membranes the monitoring system 


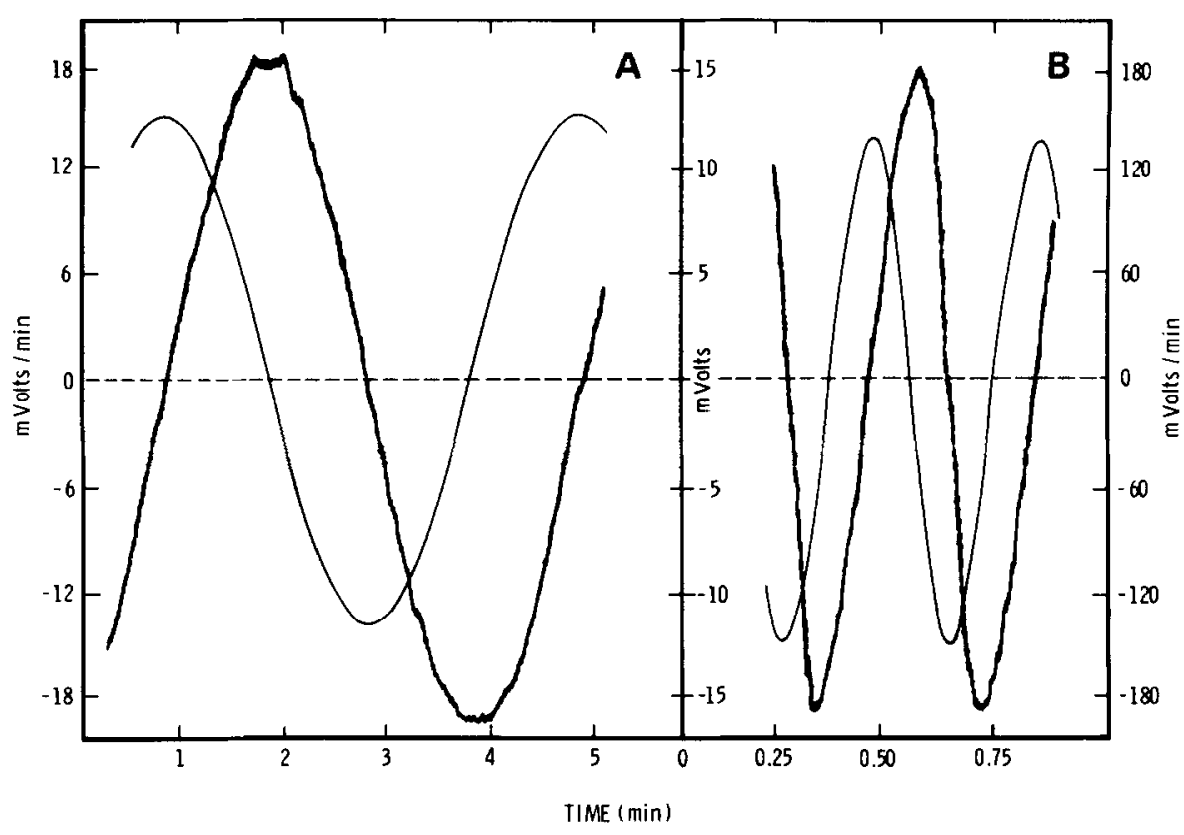

FIG. 4. Differentiator tracing of a sine wave voltage function. Sine wave voltages were produced by the function generator described under Materials and Methods (light curves), and the rates of change in voltage were displayed directly by the electronic differentiator (bold jagged curves).

could not be balanced to $100 \%$ saturation on the air scale, the $\mathrm{O}_{2}$ saturation range was used and the voltage corresponding to $100 \%$ saturation with air was noted for each membrane. This varied from $20.5 \mathrm{mV}$ with the $25-\mu \mathrm{m}$ Teflon membrane to approximately $160 \mathrm{mV}$ with the $25 \mu \mathrm{m}$ methylvinyl silicone membrane. From the differentiator tracing, the rate of change in the oxygen concentration detected by the electrode over a period of time was determined for each of the membrane materials and was plotted as shown in Fig. 5. A characteristic first-order transport constant for each type of membrane was determined from the slope of semilog plots and the values are summarized in Table 3. In accord with the different transport constants, the time required before

TABLE 2

Relationship between Oxygen Concentration and Voltage ${ }^{a}$

\begin{tabular}{|c|c|c|c|}
\hline Reaction buffer & $\begin{array}{c}\text { Catalase } \\
\text { (350 units } / \mathrm{ml} \text { ) }\end{array}$ & $\mu \mathrm{M}$ NADH oxidized $/ \mathrm{mV}$ & $\mu \mathrm{M} \mathrm{O} / \mathrm{mV}$ \\
\hline $0.1 \mathrm{M}$ Tris-HCl, pH 8.5 & + & $4.66 \pm 0.12$ & $2.33 \pm 0.06(21)$ \\
\hline 0.1 м Tris $-\mathrm{HCl}, \mathrm{pH} 8.5$ & - & $2.36 \pm 0.04$ & $2.36 \pm 0.04(7)$ \\
\hline 0.1 м Borate, pH 9.5 & + & $4.20 \pm 0.08$ & $2.10 \pm 0.04(3)$ \\
\hline 0.1 M Borate, pH 9.5 & - & $2.56 \pm 0.07$ & $2.56 \pm 0.07(4)$ \\
\hline Average & & & $2.34 \pm 0.06(35)$ \\
\hline
\end{tabular}

a Calibration assays were performed in $3.0 \mathrm{ml}$ of buffer according to the method described by Robinson and Cooper (15). NADH concentration was determined by weight and by calculation from the absorbance at $339 \mathrm{~nm}$ using a $\epsilon=6220$ liters $\mathrm{mol}^{-1} \mathrm{~cm}^{-1}$. NADH was totally oxidized during the reaction. Results are the means \pm standard errors for the number of experiments shown in parentheses. 


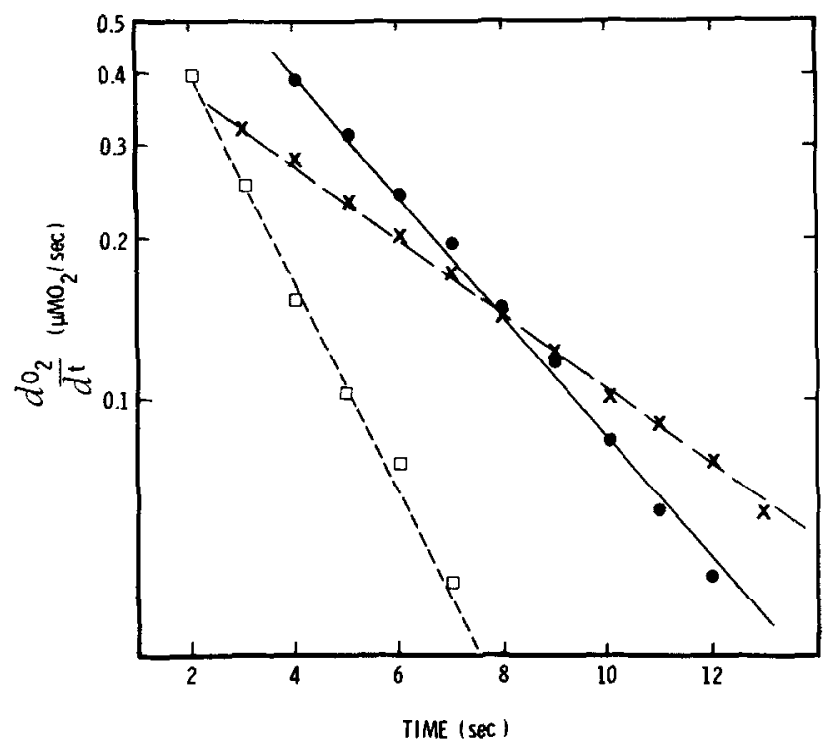

FIG. 5. Response to difference in oxygen concentration using different membranes on the oxygen electrode. The oxygen concentration was reduced by $3.5,7.0$, or $14.0 \mu \mathrm{M} \mathrm{O}$ by rapidly introducing deaerated buffer into the electrode chamber. The electrode and differentiator responses were monitored as described in the text. The rate of change in oxygen concentration, determined from the differentiator tracing, was plotted versus time for cach of the membrane materials. $0,25 \mu \mathrm{m}$ Teflon; $\square, 25 \mu \mathrm{m}$ dimethylvinyl silicone; X, $50 \mu \mathrm{m}$ dimethylvinyl silicone.

equilibrium was reached (and the changed response of the electrode accurately reflected the changed oxygen concentration) was also a characteristic of the membrane material and thickness. A 4.4- $\mu$ m-thick dimethyl silicone film could not be used with our oxygen electrode system, possibly due to its extremely high permeability to $\mathrm{O}_{2}(16)$.

\section{DISCUSSION}

The electronic differentiator is very useful in decreasing the time needed to acquire a value for a reaction velocity and in increasing the reliability and accuracy of determined velocities. For example, in our studies of the oxygenation of fatty acid substrates by

TABLE 3

Comparison of Different Membranes Used on the Oxygen-Sensing Electrodes ${ }^{a}$

\begin{tabular}{ccccc}
\hline Membrane tested & $\begin{array}{c}\text { Thickness } \\
(\mu \mathrm{m})\end{array}$ & $n$ & $\begin{array}{c}\text { Oxygen } \\
\text { transport constant } \\
\left(\mathrm{s}^{-1}\right)\end{array}$ & $\begin{array}{c}\text { Time to reach } \\
\text { equilibrium } \\
\text { (s) }\end{array}$ \\
\hline Teflon (Dupont) & 25 & 17 & $0.24 \pm 0.04$ & $21 \pm 4$ \\
Dimethylvinylsilicone & 12.5 & 9 & $0.5 \pm 0.1$ & $17 \pm 4$ \\
(Quantum, Inc.) & 50 & 5 & $0.16 \pm 0.01$ & $32 \pm 13$ \\
& 25 & 9 & $0.5 \pm 0.1$ & $17 \pm 2$ \\
& 12.5 & 6 & $>0.65$ & $6 \pm 2$ \\
\hline
\end{tabular}

a The first-order, oxygen transport constant for each membrane was determined from the slope of semilog plots as described for Fig. 5. The time to reach equilibrium corresponded to the time when the signal-to-noise ratio became less than $2: 1$ (i.e., about $0.01 \mu \mathrm{M} \mathrm{O}$ /s); this equilibrium was reached in approximately 10 half-lives. 
prostaglandin cyclooxygenase $(10,11)$ and by soybean lipoxygenase (14), the velocities continuously changed during the reaction and hundreds of velocity values were automatically displayed directly. During those studies it became evident that monitoring rapid changes of oxygen levels using the oxygen-sensing electrode was not providing an instantaneous reflection of the enzyme activity within the reaction chamber and that the inclusion of a factor to account for the membrane characteristics was requircd for precise quantitative interpretation of the oxygenase activity. This was particularly true when we attempted to compare experimental data in which rapid changes in oxygen concentration were being monitored with values generated by a computer using the enzyme kinetic model.

For example, the velocities of lipoxy- genase observed at early time points with varying added levels of product-activator (14) were considerably lower than those predicted theoretically until the membrane transport constant of $0.25^{-1}$ was also included in the model theory (Fig. 6). Similarly, this same factor (characteristic of the $25-\mu \mathrm{m}$ Teflon membrane being used) was also necessary in our evaluation of the rapid kinetic aspects of the inhibition of prostaglandin cyclooxygenase by drugs (11).

Thus, we have found that the improvements in quantitation of the electrode response afforded by the electronic integrator and the modifications of membrane response of the electrode greatly extend the sensitivity and versatility of the biological oxygen monitor as an analytical tool. This is particularly true for application in continuous evaluation of changes in reaction rates of

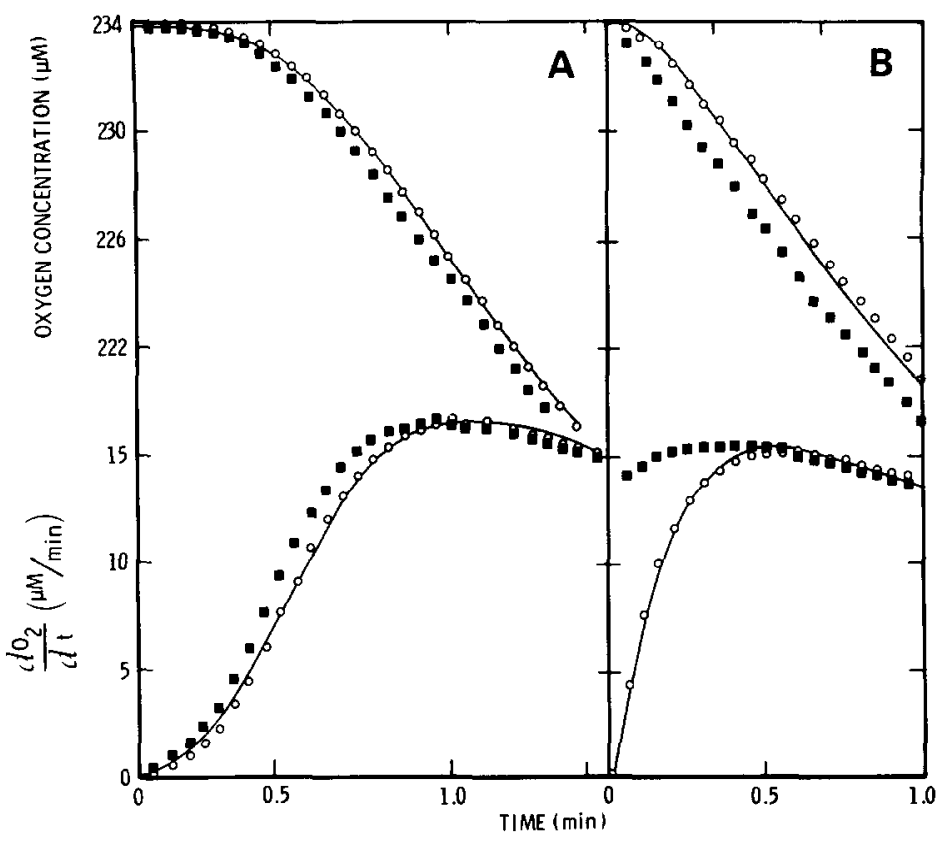

FIG. 6. Evaluation of an oxygenase reaction system using the electronic differentiator. Oxygenation of $100 \mu \mathrm{M} \mathrm{5,8,11,14-eicosatetraenoic} \mathrm{acid} \mathrm{by} 1 \mu \mathrm{g}$ of soybean lipoxygenase in $3.0 \mathrm{ml}$ of $0.1 \mathrm{M}$ Tris- $\mathrm{HCl}$ buffer ( $\mathrm{pH} 8.5$ ) was monitored with an oxygen electrode covered with $25-\mu \mathrm{m}$ Teflon membrane. The concentrations of product-activator present initially were: (A) 0.05 and (B) $4.0 \mu \mathrm{M}$. The upper lines represent the oxygen concentration monitored by the electrode and the lower lines represent the recording from the electronic differentiator. Both reaction mixtures were simulated using a computerized model for lipoxygenase and the values for kinetic constants described previously (Ref. 14). The model included an adjustment for oxygen transport through the electrode membrane; calculated values are shown as discrete points for rate constants of $0.24(O)$ and $1.0 \mathrm{~s}^{-1}(\square)$. 
oxygen consuming or producing enzymes. With the information provided, adaptations of commercially available components can be made with assistance from most versatile electronics shops. The differentiator described gives a continuous recording of instantaneous velocity without requiring time consuming and partially subjective estimates using tangential lines made by the experimenter. The improved kinetic monitoring of reaction rates with the differentiator can be extended further to experiments in which absorbance is monitored spectrophotometrically or $\mathrm{pH}$ changes are monitored with a $\mathrm{pH}$ meter. In each case the output voltage of the monitoring instrument (usually $0.1-1.0 \mathrm{~V}$ ) can be filtered smooth and differentiated electronically to give a direct rate of reaction as described above for the oxygen electrode. We have found that detailed direct display of reaction velocity in an irreplacable tool in evaluation of complex kinetic responses.

\section{ACKNOWLEDGMENTS}

The authors are grateful to Dr. R. L. Riley for supplying the dimethyl silicone membrane tested. This work was supported in part by Grant GB-28663X from the National Science Foundation and a Medical Research Council of Canada fellowship (H.W.C.).

\section{REFERENCES}

1. Clark, L. C. (1956) Trans. Amer. Soc. Art. Int. Organs 2, 41-48.

2. Fatt, I. (1976) Pularugraphic Oxygen Sensors, Chem. Rubber Co., Cleveland.

3. Penniston, J. T. (1972) Arch. Biochem. Biophys. 150, 556-565.

4. Trudgill, P. W. (1974) Anal. Biochem. 58, 183-189.

5. Luzzana, M. R., and Penniston, J. T. (1975) Biochim. Biophys. Acta 376, 157-164.

6. Muenzer, J., Weinbach, E. C., and Wolfe, S. M. (1975) Biochim. Biophys. Acta 376, 237-242.

7. Halbach, S. (1977) Anal. Biochem. 80, 383-391.

8. Del Rió. L. A., Ortega, M. G., López, A. L., and Gorgé, J. L. (1977) Anal. Biochem. 80, 409-415.

9. Smith, W. L., and Lands, W. E. M. (1972) Biochemistry 11, 3276-3285.

10. Cook. H. W., and Lands, W. E. M. (1975) Biochem. Biophys. Res. Commun. 65, 464-471.

11. Lands, W. E. M., Cook, H. W., and Rome, L. H. (1976) In Advances in Prostaglandin and Thromboxane Research (Samuelsson, B., and Paoletti, R., eds.), Vol. 1, pp. 7-17, Raven Press, New York.

12. Cook. H. W., and Lands, W. E. M. (1976) Nature (London) 260, 630-632.

13. Smith, W. L., and Lands, W. E. M. (1972) J. Biol. Chem. 247, 1038-1047.

14. Cook. H. W., and Lands, W. E. M. (1975) Canad. J. Biochem. 53, 1220-1231.

15. Robinson, J., and Cooper, J. M. (1970) Anal. Biochem. 33, 390-399.

16. Reardon, J. D., and Doede, D. R. (1975) Indust. Res. 17, 52-55. 